\title{
STRUCTURALLY CONSTRAINED AERODYNAMIC ADJOINT OPTIMISATION OF HIGHLY LOADED COMPRESSOR BLADES
}

\author{
Cleopatra Cuciumita ${ }^{1}$, Alistair John ${ }^{1}$, Ning Qin ${ }^{1}$, Shahrokh Shahpar ${ }^{2}$ \\ 1 Department of Mechanical Engineering, University of Sheffield, Sheffield, S1 3JD, United Kingdom \\ 2 Innovation Hub, Central Technology, Future Methods, Rolls-Royce plc., Derby, DE24 8BJ, United Kingdom
}

\begin{abstract}
Adjoint aerodynamic optimisation has recently gained increased popularity for turbomachinery applications due to the large number of parameters that can be used without incurring additional major computational costs. This work presents an adjoint based aero-structural optimisation method having efficiency as the objective function and maximum von Mises stress set as a constraint. The full optimisation loop was set up with free-form deformation for geometry parametrisation. A response surface was created beforehand for computing the maximum von Mises stress using a meshless method. A discrete adjoint approach was used to obtain the gradients of the objective function with respect to each design parameter, while the constraint gradients were computed using finite differences. A sequential least squares programming algorithm was used as the optimizer. Tests carried out on a highly loaded compressor blade showed that the method successfully increases the efficiency by more than 3\% while maintaining the maximum stress under the imposed value. The results also showed that the constrained optimisation loses about $1 \%$ in potential efficiency gain compared to the same optimisation process without stress constraint. Overall, the work provides a methodology for conducting structurally constrained adjoint aerodynamic optimisation that can be applied for large number of design parameters while maintaining low computational costs. It also provides reference for constructing and selecting a response surface to be used in the optimisation process.
\end{abstract}

Keywords: adjoint, optimisation, free-form deformation, von Mises stress, constraint

\section{NOMENCLATURE}

CFD Computational Fluid Dynamics

$\begin{array}{ll}\text { DoE } & \text { Design of Experiments } \\ \text { FEM } & \text { Finite Element Method } \\ \text { FFD } & \text { Free-Form Deformation } \\ \text { LH } & \text { Latin Hypercube } \\ \text { R }^{2} & \text { Coefficient of determination } \\ \text { RMSE } & \text { Root-mean-square error } \\ \text { RSM } & \text { Response Surface Method } \\ \text { SLSQP } & \text { Sequential Least SQuares Programming }\end{array}$

\section{INTRODUCTION}

\subsection{Motivation}

Designing highly loaded transonic compressor blades is a challenging task. Considering today's competitive market, the design requirements are not only driven by several disciplines like aerodynamics, structural mechanics and acoustics, to be more efficient, reliable and quieter, but they are also subjected to lower costs and much shorter design time scales. The classical design approach is an iterative process between the different disciplines until all requirements are satisfied. However, a shift in paradigm is currently rising, with the development of advanced optimisation methods to achieve these requirements. The focus has become multidisciplinary optimisation, which aims at taking into account disciplinary interactions during the design process.

The objective of this work is to provide an optimisation methodology for highly loaded compressor blades to increase their efficiency while maintaining an acceptable value of maximum stress. The novelty of the methodology relies on coupling an adjoint-based aerodynamic optimisation, which can be applied for a large number of design parameters without introducing additional computational costs, with a response surface based model for constraining the maximum stress on the blade. This higher fidelity structural analysis negates the 
need to use limiting pedigree rules during the aerodynamic optimisation.

First, a survey of previous work relevant to this paper is presented, with respect to the optimisation case studied, the geometry deformation method and the adjoint optimisation method. Next, the methods and tools used in this study are described for: the mesh generation and CFD setup, the geometry parametrisation, the adjoint method used and the stress analysis setup. A description of the response surface generation and selection of the response surface method follows. An overview of the optimisation process is also given. The optimisation results are presented and the optimal geometry is discussed from both an aerodynamic and structural standpoint, before summarising the conclusions.

\subsection{Previous work}

\subsubsection{Case studied - NASA Rotor 37}

Much of the progress in the field of optimisation methods for the design of transonic compressor blades was achieved based on the publicly available transonic compressor NASA rotor 37 [1], and research using Rotor 37 covers over $75 \%$ of the publications in this field [2].

In-depth analysis of previous optimisations studies conducted on this test case were documented in [2] and [3]. The authors show the wide range of methods explored in the literature, evolving from simple, single-objective, unconstrained optimisation problems with only three design parameters [4] to multi-objective [5], constrained [5-7], higher number of variables [7] optimisation methods.

The optimisation methods used vary with the evolution of optimisation algorithms in general, and cover both high and low fidelity analyses. This includes evolutionary algorithms [5], metamodeling approaches $[6,7]$ or, more recently, adjointbased methods [8]. Most of the blade parametrisations were done using either engineering parameters or camber and thickness distribution [5-8], with some work being done using free-form deformation $[2,9]$. The large variety of optimisation methods and number of optimisation parameters also results in a great variety of incurred computational costs.

From the early work in the filed it was clear that an unconstrained optimisation leads to solutions with increased efficiency but decreased rotor pressure ratios [4]. Therefore, further efforts focused on constraining the pressure ratio [6-8]. However, most of the constraints were purely aerodynamic, referring either to the pressure ratio or mass flow rate. Yet some of these studies $[5,7]$ showed that purely aerodynamic optimisations resulted in highly leant geometries, which would most likely be mechanically unacceptable. To mitigate this, efforts were made by some authors towards a multidisciplinary approach, by introducing the structural mechanics discipline as a constraint, either in the form of maximum stress [6] or minimum thickness [9]. This resulted in an optimised shape of the blades having lower efficiency, but with no damaging impact on the structural integrity.
Overall, these previous results provide a useful insight into the efficiency benefits that can be achieved through optimisation for comparison purposes. The average efficiency benefit achieved was in the range of $1.7-1.9 \%$, without any structural constraints, with a maximum value of $3.5 \%$ benefit reported in [9], when using adjoint-based optimisation and free form deformation parametrisation. In the multidisciplinary approaches, the reported efficiency benefit dropped to values in the range of $1.0-1.5 \%[6,9]$.

\subsubsection{Free Form Deformation for turbomachinery}

Free-form deformation is nowadays the most widely used of the volumetric deformation methods. Introduced in 1986 [10], it is based on encompassing the solid shape by a trivariate Bezier volume. The control points of the volume represent the design parameters and they are directly linked to the solid shape. A one-to-one correspondence between the points within the original and deformed volumes is established. Changing the position of these points leads to a deformation of the geometry inside the volume, while preserving the topology.

FFD has the main advantage over other design parameters in that it provides a better shaping flexibility and, thus, greater design potential. On the other hand, as the control points have no apparent physical meaning, conclusions relating them to the performances are difficult to interpret and not intuitive.

FFD has been successfully used in turbomachinery optimisation problems previously, either to increase the efficiency of a turbine $[11,12]$ or to reduce the shock effects on the performances of an open rotor [13]. A recent application of an FFD approach for the shape optimisation of a transonic compressor was shown in [2]. The authors concluded that their FFD with 54 points parametrisation produced an optimised sshaped geometry of increased benefit compared to the literature, by weakening the passage shock and significantly reducing the shock-induced separation.

The FFD geometry parameterisation technique was shown to produce a design of increased benefit compared to that previously found in the literature. In a subsequent study [9] they have shown that FFD can lead to unfeasible designs and some sort of structural constraint is needed, such as thickness constraint, although this reduces the efficiency benefit, from $3.5 \%$ to $2.5 \%$. They have also shown that increasing the number of FFD parameters in the axial and circumferential directions is key to increasing the performances, but the optimisation results plateau beyond a resolution of $4 \times 4$. They also showed that increasing the radial resolution actually proved detrimental for rotor 37 as increasing the radial control sections may make it harder for the optimiser to find an optimal design.

\subsubsection{Adjoint optimisation of turbomachinery}

While FFD has been proven to have potential for higher efficiency benefit when used in turbomachinery optimisation, it 
can lead to a large number of parameters (273 parameters for the turbine optimisation conducted in [11]). For this reason, it lends itself to adjoint-based shape optimisation.

The adjoint method has the major benefit that it computes the gradients of an objective function independent of the number of variables. In the aeronautical field, the use of adjoint method was pioneered by Jameson [14] and has been gaining increased momentum in the last couple of decades.

In turbomachinery optimisation applications, adjoint optimisation work focuses mainly on aerodynamic cost functions and constraints [8, 15 - 22]. As the importance of including the structural integrity within the design and optimisation process became more apparent, work on turbomachinery adjoint structural optimisation to reduce the maximum stress has recently emerged, with a focus on radial turbines [23, 24]. This was followed by a multidisciplinary approach involving aerodynamics and structural analysis and using decoupled adjoint optimisation for improving the efficiency of radial turbines either at design conditions [25] or at two operating points $[26,27]$ while constraining the maximum von Mises stress [25, 26] or its moment of inertia [27].

For transonic compressors in particular, optimisation work using adjoint methods is scarce. Out of the existing work, such as $[8,9,28]$, the highest efficiency benefit was obtained by combining adjoint optimisation with FFD parametrisation in [9]. For this reason, this combination was chosen for this work as well. With the exception of the thickness constraint imposed in [9], no published work on transonic compressors adjoint optimisation considering structural integrity was found in the literature. This paper builds on the previous work done in [9] and takes it a step further, by adding a structural constraint in the optimisation process in the form of the maximum von Mises stress.

\section{METHODS AND TOOLS}

For the aero-mechanical optimisation of a highly loaded compressor blade, the NASA Rotor 37 geometry was selected as case study. FFD parametrisation, coupled with adjoint aerodynamic optimisation was used. The objective function was efficiency. A structural constraint for the maximum equivalent (von Mises) stress was also imposed. This stress was evaluated based on a response surface. A sequential least squares programming algorithm was used as the optimiser.

\subsection{Case studied - NASA rotor 37}

The case selected for this investigation is NASA Rotor 37, as it is well-documented in the literature, both from an experimental standpoint, as well as with respect to optimisation results. A summary table for the main flow parameters of Rotor 37 is given in [1]. Although it dates back to the 1970, rotor 37 makes for a good test case in optimisation problems as it poses several challenges: highly separated flow, shock waves and interaction with the boundary layer and a large tip-leakage vortex. Figure 1 shows the Rotor 37 geometry, as well as the CFD setup.

The meshes were automatically generated using the inhouse software PADRAM [29], as multi-block structured grids with $\mathrm{y}+1$. Details of the mesh comprising approximately 600,000 cells are presented in Figure 2.

All the simulations presented here were conducted using the in-house Rolls-Royce code, Hydra [30]. The turbulence model used was Spalart-Allmaras. The inlet boundary conditions were specified based on the original experimental values [31] as radial distribution of total pressure and temperature while at the outlet, a value for circumferentially mixed-out and radially mass-averaged capacity was imposed. The walls are considered adiabatic viscous walls. The rotational speed was set to $1800 \mathrm{rad} / \mathrm{s}$.

The validation of the Rotor 37 datum results against experimental data from [1] using this mesh and CFD setup was reported in [9].

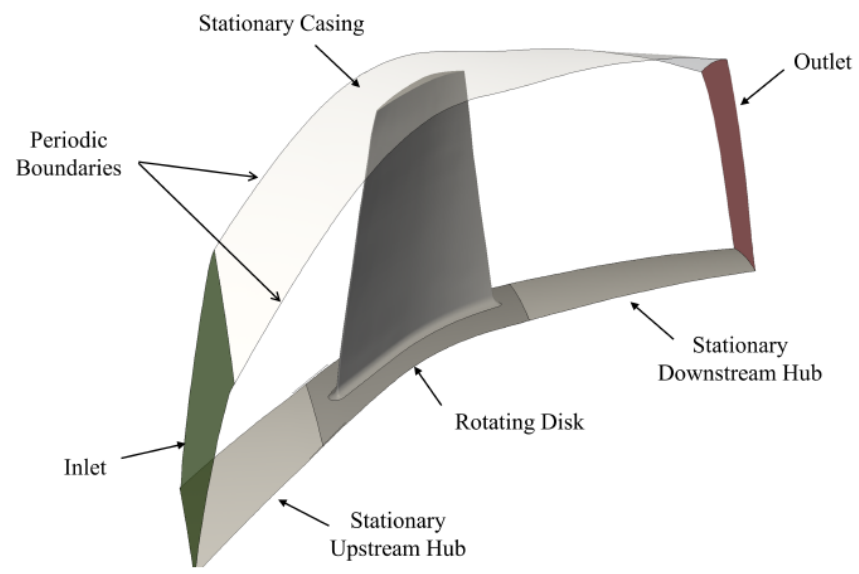

FIGURE 1: THE R37 CFD DOMAIN USED [9]

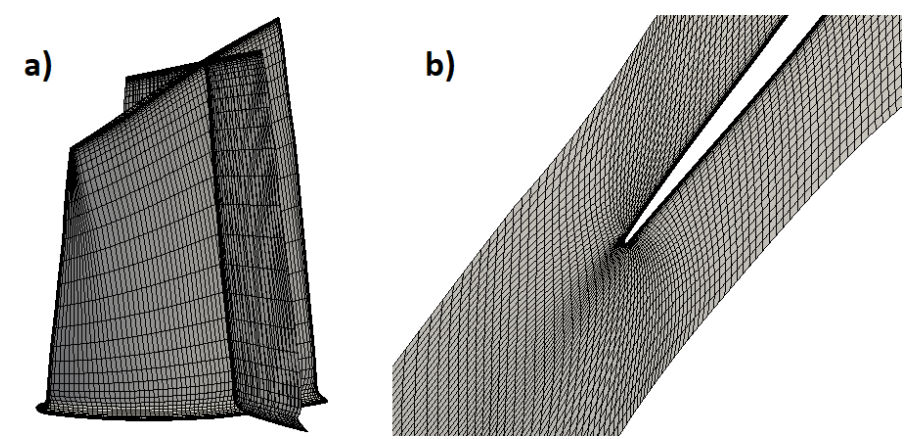

FIGURE 2: Mesh: a) Blade and axial cut b) Radial slice near leading edge

\subsection{Geometry parametrisation (FFD)}

The geometry parametrisation method follows the freeform deformation approach from [9]. The FFD 
parameterisation method is available through the geometry design capability of the in-house software PADRAM [29]. As the radial resolution of the parametrisation grid proved not to be key in the efficiency gain, and to minimize the impact of geometry morphing on the maximum stress value, the hub section was kept constant. The selected FFD grid has a dimension of $3 \times 3 \times 2$ (three axial by three circumferential FFD points, with two radial control sections). With each point having two coordinate values, it results in 36 design parameters.

Figure 3 shows the control points of the FFD grids and their movement, as well as the resultant shape. The original geometry and points are represented in orange, while the movement of the points and the resulting shape change is given in blue. It can be seen that the resulting shape change is a combination of the movement of all points that is responsible for the overall blade shaping.

There is a potential to add a thickness (or other geometric feature) constraint, as described in [9], but the purpose of this study was to test the performance of the stress constraint on its own. Valid geometries are obtained as a consequence of the stress constraint which eliminates the strongly distorted geometries. For this study, no direct geometric constraint was imposed.

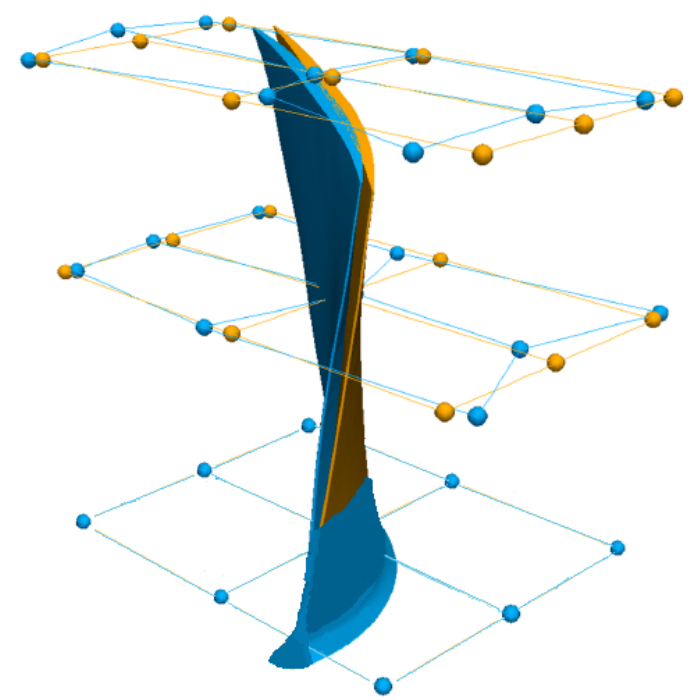

FIGURE 3: FFD GRID EXAMPLE (ORANGE - ORIGINAL, BLUE - DEFORMED)

\subsection{Adjoint method}

The adjoint method used here for computing the gradients of the objective function with respect to each design parameter and therefore the search direction is a discrete approach implemented in Hydra Adjoint [32]. The discrete adjoint solver is coupled with the primal flow solver. Both solvers use the exact same discretization method, solver procedure and solver settings to achieve fully consistent primal and adjoint solutions.
The adjoint solver involves a large linear system of equations, where the right-hand-side depends on the chosen objective. The matrix corresponds to the transpose of the Jacobian of the primal flow (i.e. the first derivative of the fluxes w.r.t. the flow variables), and the unknown variables are the adjoint vector. This means that each primal variable (e.g. density, velocity, turbulence variable) has a direct counterpart in the adjoint system of equations, i.e. there exists an adjoint density, an adjoint velocity and an adjoint turbulence variable. The Jacobian is never actually assembled, only its action on a vector is computed. The necessary first order derivatives of flux, boundary condition and objective routines are calculated by employing algorithmic differentiation of the source code.

The advantage of the adjoint approach is that it does not depend on the design parameters, but only on the objective function. This enables the possibility of computing, from a single adjoint solution, the sensitivity with respect to many design parameters.

\subsection{Stress analysis}

The stress analysis was done by fixing the hub section of the blade and considering only the centrifugal force loads for the purpose of methodology development and testing, as they are the main contributor to the stress generation for this application. Including the pressure loads will add to the accuracy of the results, but also to the computational time for generating the response surface due to the required time for transferring the loads from the CFD results to the FEM model. The result of interest, to be used as the constraint in the optimisation process, was the maximum equivalent (von Mises) stress. The material used for modelling the blade was the titanium alloy Ti-6Al-2Nb-1Ta-1Mo, with the density of 4480 $\mathrm{kg} / \mathrm{m}^{3}$, tensile and compressive yield strength of $710 \mathrm{MPa}$, and tensile ultimate strength of $830 \mathrm{MPa}$.

To further benefit from reduced computational costs, the evaluation and constraining of the maximum stress was done by using a response surface generated beforehand. To enable the use of a large number of points in the design of experiments, a fast, meshless tool was used for performing the stress analysis and building up the response surface.

SimSolid is a meshless simulation software application which performs static, dynamic and thermal analyses of structural systems. The computational algorithm is based on breakthrough extensions to the theory of external approximations [33]. External approximations are a generalization of the Finite Element Method (FEM) with regard to:

- " "finite elements" can be absolutely arbitrary shapes,

- compared to the strictly structured polynomials used in conventional FEM, basis functions which approximate field of interest in the "element" can be of arbitrary class and are independent of the "element" shape.

SimSolid controls solution accuracy using multi-pass adaptive analysis. Adaptivity can be defined on a global or part 
local basis and adaptivity is always active. Although SimSolid is new, it has been extensively tested in a variety of industries. They have produced a validation manual, available at www.simsolid.com.

The methodology is fast and efficient, allowing very large and/or complex assemblies to be solved quickly on desktop class computers. Although there is an argument in the favour of using this tool directly in the optimisation framework, the uniqueness of this software would hinder the reproducibility of the results by other, higher fidelity, structural tools, if this particular software is unavailable. At the same time, as this software is currently Windows based, without the possibility of running in batch mode, hence using it in an optimisation process is not possible. With the available mesh, CFD and adjoint codes there are also issues of portability and integration, outside the scope of this work. For these reasons, it was opted to make the structural assessment based on a response surface method.

The von Mises stress distribution computed with SimSolid for the datum geometry is given in Figure 4 (top). A verification for the value of the maximum von Mises stress obtained with SimSolid for the datum geometry was conducted by comparing it against the results obtained using a wellestablished, traditional FEA tool. The structural analysis was done using Ansys Mechanical under Ansys Workbench 19.2 using the same structural model and material properties. To ensure that the solution is mesh independent, the fully automatic adaptive mesh refinement option integrated into the Ansys solution process was used [34]. The results have converged within an accuracy of $0.1 \%$ and are plotted in Figure 4 (bottom). Figure 4 shows that a good agreement between results is obtained, with a relative difference of $2.2 \%$ between the maximum value of the von Mises stress computed with SimSolid (243.8 MPa) and that computed with Ansys Mechanical (249.3 MPa).

The use of SimSolid for running the stress analysis showed more than tenfold decrease in computational time compared to Ansys Mechanical. This allows for faster response surface generation, or the creation of a more accurate response surface in the same amount of time, based on a larger number of points in the design of experiment.

\subsection{Stress Response Surface}

For the maximum von Mises stress response surface generation, two design of experiments were generated using the Latin Hypercube sampling method, using 2000 points and 20000 points, respectively. The smaller number of points was first selected for computational time reasons and then increased to reach a value of the determination coefficient of over 0.9 .

The maximum von Mises stress values was computed for all of the geometries in the two design of experiments and used to generate response surfaces based on three different methods: polynomial, Kriging and radial basis function methods. This resulted in six different response surfaces. The process used for generating the stress response surfaces is described in Figure 5.
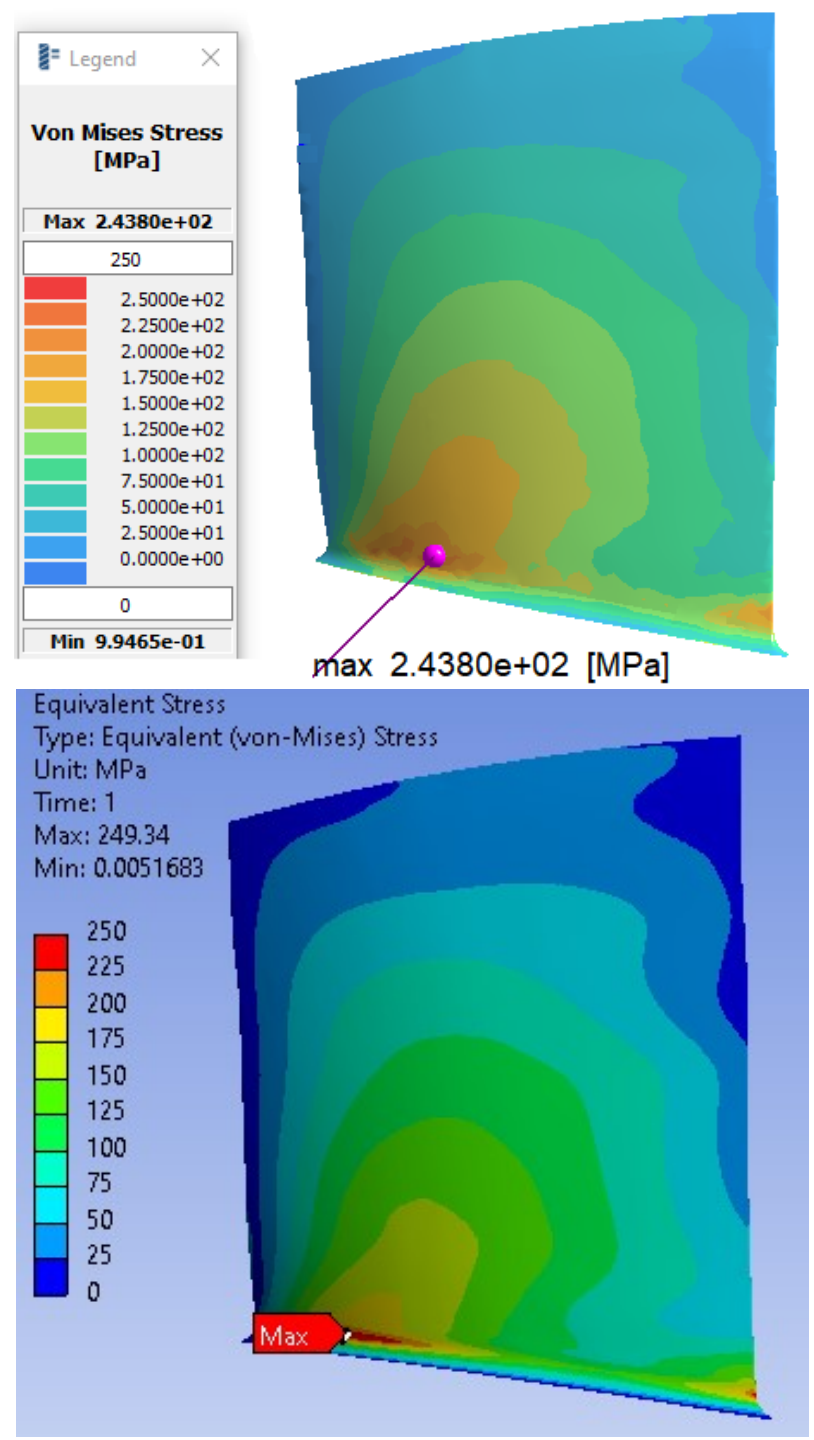

FIGURE 4: R37 VON MISES STRESS WITH SIMSOLID (TOP) AND ANSYS MECHANICAL (BOTTOM)

RSM generation

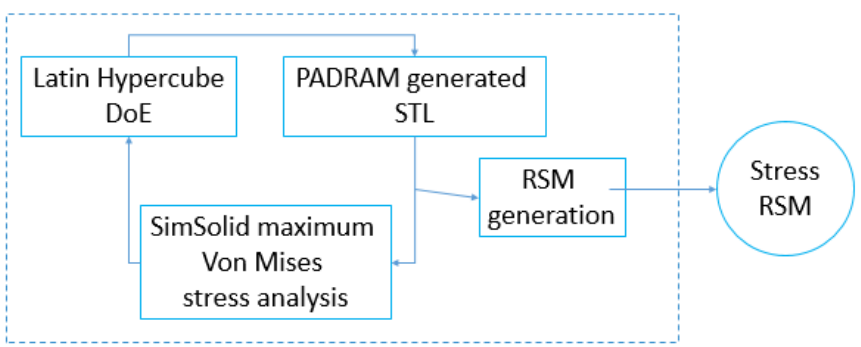

FIGURE 5: WORKFLOW DIAGRAM FOR THE STRESS RSM 
A comparison of the six response surfaces generated was done based on the coefficient of determination, the root mean square error and computational time.

The coefficient of determination is the square of the Pearson's coefficient (i.e., $\mathrm{R}^{2}=\mathrm{r}^{2}$ ) and describes the proportion of the total variance in the observed data that can be explained by the model. It provides a measure of how well observed values are predicted by the model and it ranges from 0.0 to 1.0 , with higher values indicating better agreement.

The Root-Mean-Square-Error (RMSE) measures the differences between values predicted by the model and the observed values. It is the average vertical distance of the actual data points from the fitted line. Smaller RMSE reflects greater accuracy.

Both the RMSE and coefficient of determination $\left(\mathrm{R}^{2}\right)$ offer different, yet complementary, information. The RMSE gives a sense of how close the predicted values are from the actual data. It is relatively easy to understand and communicate since reported values are in the same units as the dependant variable being modelled. The effect of each error on RMSE is proportional to the size of the squared error, which makes it sensitive to large errors (penalizes large prediction errors more than smaller prediction errors).

The $\mathrm{R}^{2}$ gives an overall sense of how well the predicted values fit the data. Statistical texts frequently illustrate that $R^{2}$ is sensitive to outliners [35]. A model that can follow the observed data during extreme events, i.e., located far away from the others, will have an artificially higher value of $\mathrm{R}^{2}$, which may obscure the true relationship between the predicted and observed data over most of the remainder of the domain.

Two different sampling methods of the same design space were used to assess the six response surfaces and compare them.

Sampling 1 was done based on 200 geometries generated by the Latin Hypercube method.

A stress analysis was conducted for each of these geometries to compute the actual maximum von Mises stress and compare it with the predicted values coming from each response surface method. The corresponding $\mathrm{R}^{2}$ and RMSE are plotted in Figure 6, for all RSM generated. The data is plotted from left to right to reflect the response surfaces from best to worse, according to each of the two metrics. Although the values are very close for the first three RSMs, the two criteria display different ranking. To make sure the above described pitfall with respect to outliners is avoided, a scatter plot to visually inspect it for outliers is depicted in Figure 7. This plot shows the presence of two points (for each of the response surface) located further then the cluster data, which were eliminated. The RMSE and $\mathrm{R}^{2}$ values were recomputed without their data. The new values are given in Figure 8. Figure 8 shows a good correlation between the RMSE and $\mathrm{R}^{2}$ ranking, and places first the polynomial RSM generated with 20000 design points, followed by the RBF and the Kriging methods with same number of points.

Sampling 2 consisted of 30 geometries that were generated in an unconstrained optimisation process.

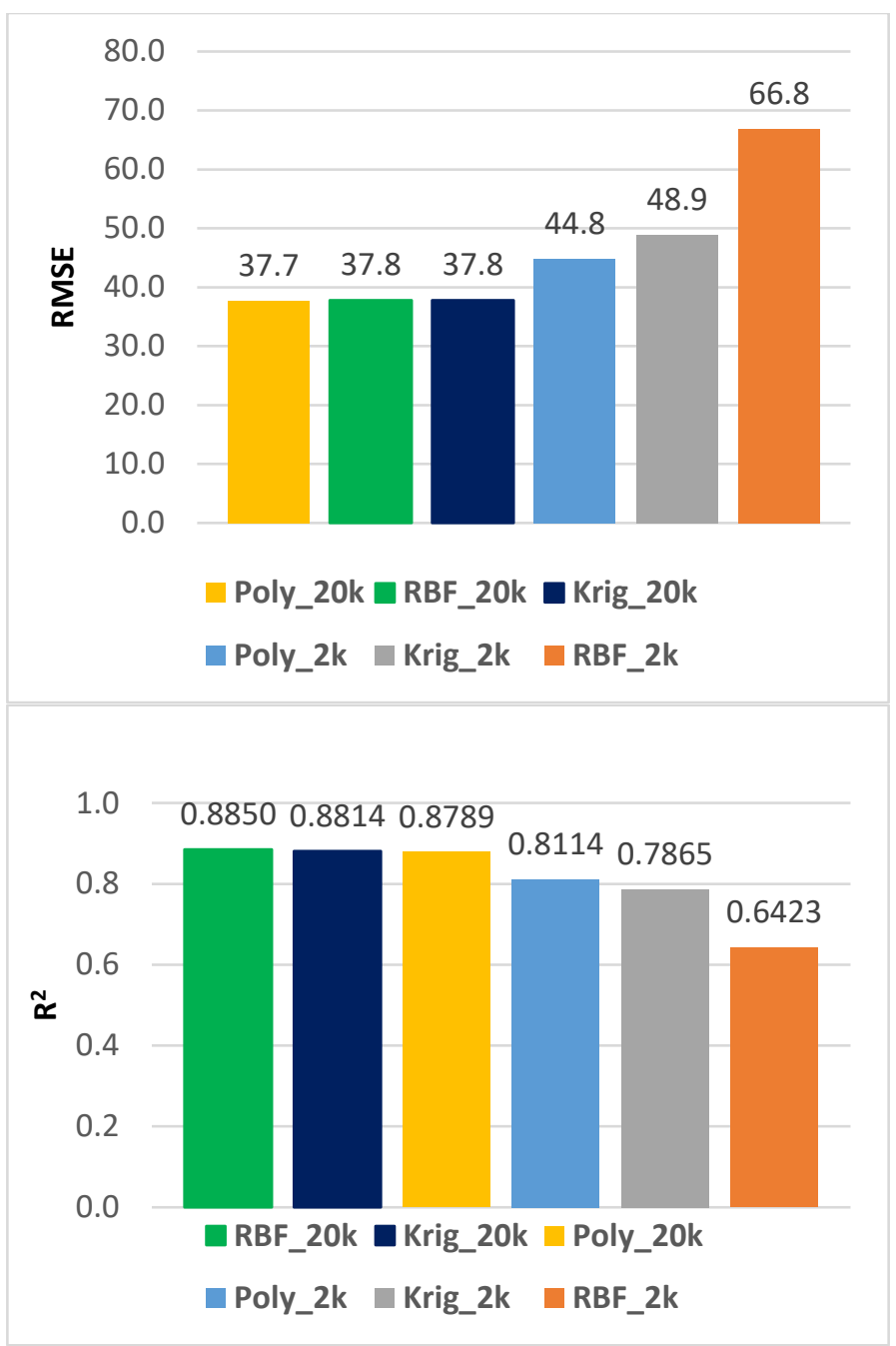

FIGURE 6: SAMPLING 1 RSM COMPARISON

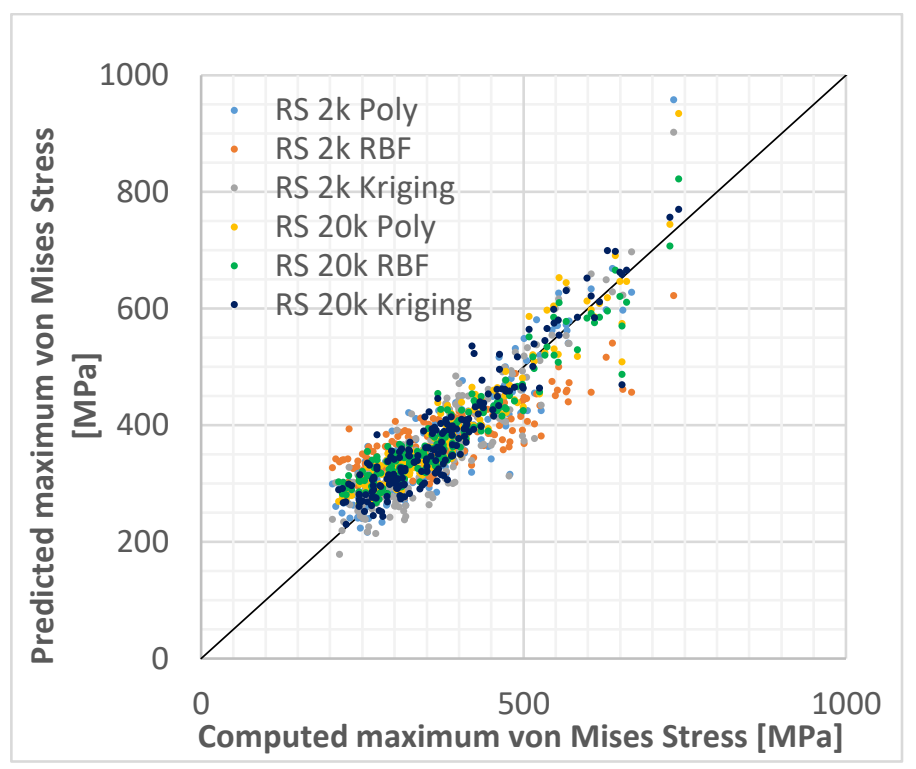

FIGURE 7: SAMPLING 1 RSM COMPARISON 


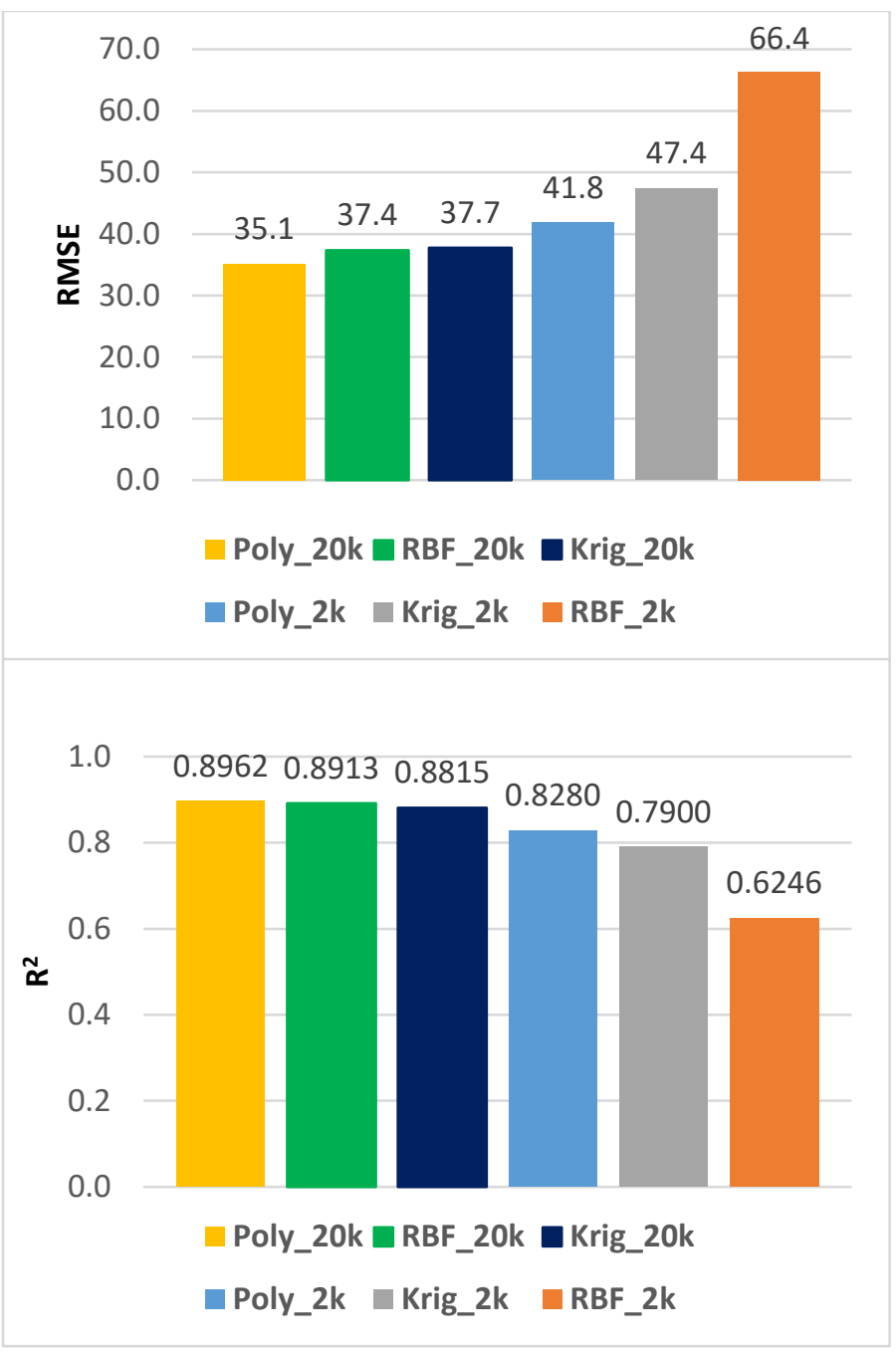

FIGURE 8: SAMPLING 1 RSM COMPARISON

The purpose of this sampling was to test the accuracy of the response surface in the region of interest for improving the efficiency. As with the previous sampling, the data was visualised for potential outliners, see Figure 9.

The point furthest from the main set of data was considered an outliner and eliminated from processing the data. The RMSE and $\mathrm{R}^{2}$ values computed without this point data are given in Figure 10, for comparison and ranking of the response surfaces. The results of this sampling support the results of sampling 1, also pointing to the polynomial RSM based on 20000 points as giving the best results.

Since the computational time is dependent on the number of points in the DoE, two different sets of DoEs were considered (with 2000 and 20000 points, respectively), in case the larger one proved prohibitive from this standpoint. The time required to compute the maximum stress values increased tenfold, proportionally with the number of points in the DoE, from three days to approximately four weeks.

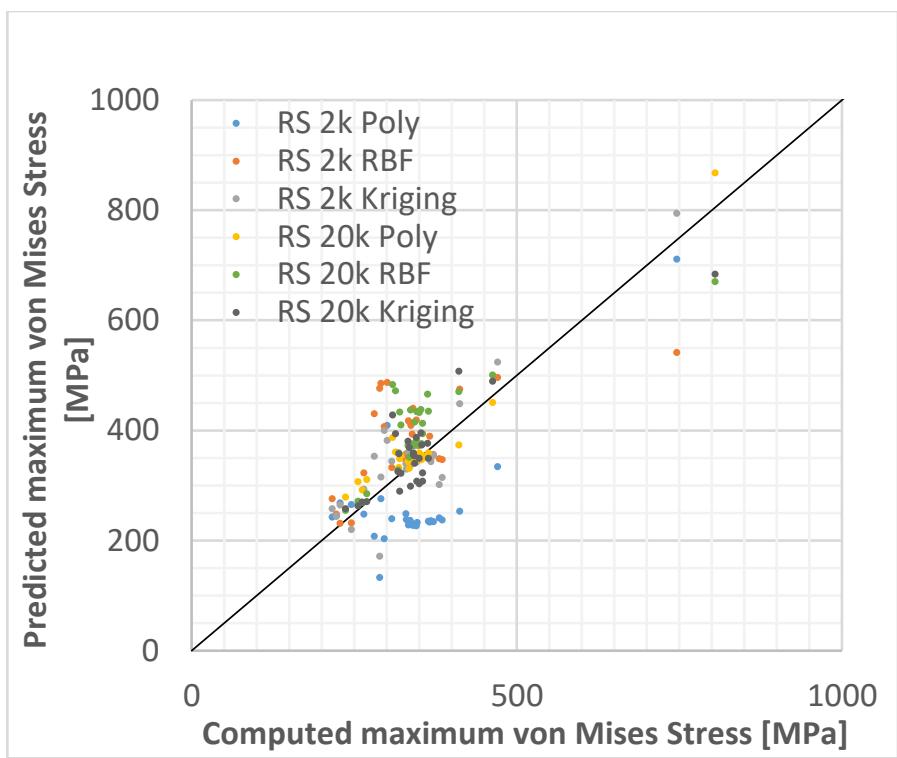

FIGURE 9: SAMPLING 2 RSM COMPARISON

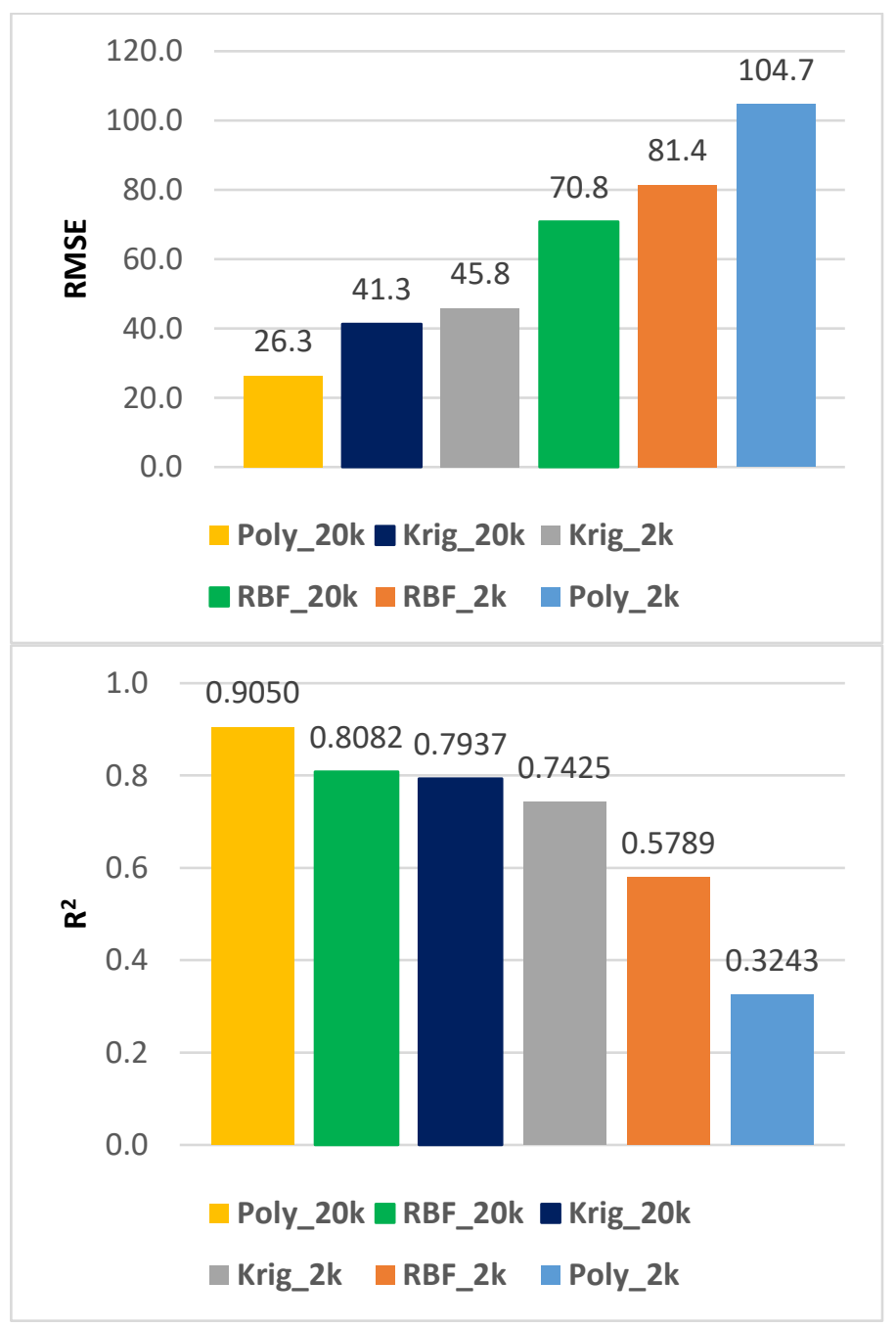

FIGURE 10: SAMPLING 2 RSM COMPARISON 
In terms of the time required to generate the response surface based on the data gathered, the computational time increased from minutes to a few hours. The only one that proved particularly expensive in terms of computational costs was the Kriging approach with 20000 points. This data can be used as guideline for the computational time required when generating other response surfaces based on this method.

Based on all of the above, for this study, the polynomial RSM based on 20000 points was further used as a metamodel in the structurally constrained aerodynamic adjoint optimisation of the highly loaded compressor blades.

\subsection{Optimisation process}

For the optimisation process, a Sequential Least SQuares Programming (SLSQP) [36] algorithm is used in this work. This algorithm uses the Han-Powell quasi-Newton method with a BFGS update of the B-matrix and an L1 test function in the step length algorithm. The optimizer uses a slightly modified version of Lawson and Hanson's NNLS nonlinear least-squares solver.

The process starts with the initial design, or datum geometry. Using the adjoint process, the objective function (efficiency) and gradients with respect to each design parameter are computed. The stress and corresponding gradients are calculated based on the response surface previously generated and finite differences.

The optimizer then selects a feasible direction. To estimate the objective function behaviour in this direction, various step sizes are used for computing the objective function, the minimum of which is further used. This is considered the new optimal design, and the process is repeated to obtain a new set of gradients and a search direction.

The optimisation steps used in this work, in brief, are:

- First, the stress response surface is generated separately, using the same design space as the one used in the optimisation process

- Using the current set of design parameters, the geometry for the current step is generated and meshed in PADRAM

- CFD pre-processing and multi-grid generation is applied

- The flow is solved to calculate the objective function value for the current geometry using Hydra

- The gradients between the objective function and the flow field, and the flow field and the mesh nodes are computed in Hydra Adjoint while finite difference is used to calculate the gradient of the mesh nodes to each design parameter

- Using the generated response surface, the maximum stress is computed for the current geometry and the stress gradients are determined by finite difference for each design parameter

- A feasible direction is obtained by solving the quadratic programming problem and used in a line search to select the next design to be run

- Several designs are run (without adjoint) until a new optimal point is found
- The process is then repeated until a convergence is achieved for the objective function.

The resulting workflow diagram is depicted below, in Figure 11.

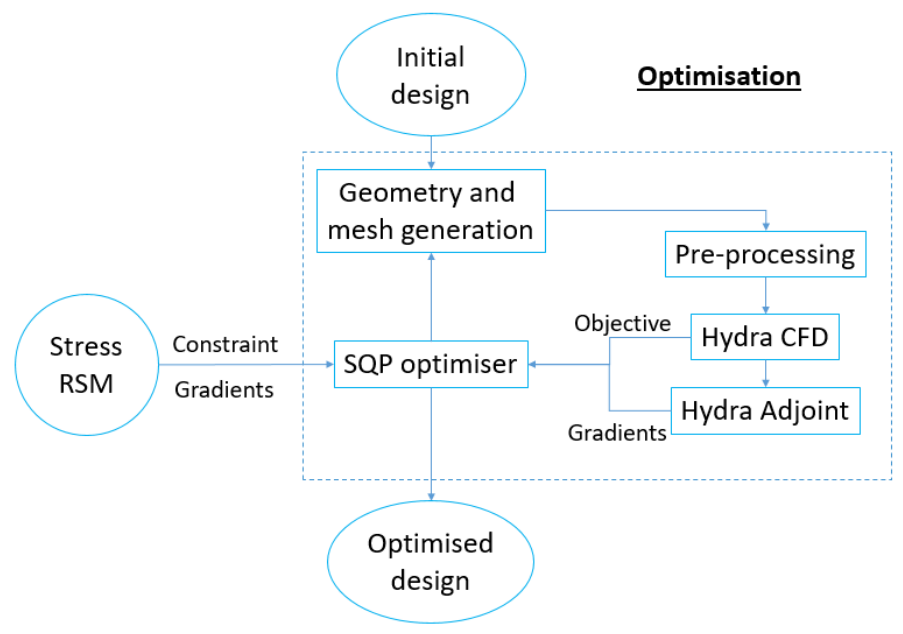

FIGURE 11: WORKFLOW DIAGRAM FOR THE CONSTRAINED ADJOINT OPTIMISATION PROCESS

\section{RESULTS AND DISCUSSION}

\subsection{Optimisation results}

Two different optimisation runs were carried out. The first optimisation was done without any structural constraint, for comparison purposes. The second optimisation was conducted with a structural constraint based on the algorithm described above, for the maximum von Mises stress constrained to the datum value.

The evolution of the efficiency (objective function) and maximum stress (constraint), during both constrained and unconstrained optimisation runs, are depicted in Figure 12.

The results show that the maximum stress was successfully constrained to the datum value, but with a toll on the efficiency benefit, as compared to the unconstrained optimisation results. This result is expected when constraining the optimisation process, and is a direct consequence of reducing the range and flexibility of the designs that can be produced in the given design space. As the optimisation is based on FFD, rather than engineering parameters, it is difficult to assess which geometrical feature has been limited more in the constrained optimisation compared to the unconstrained one. For this reason, a discussion on the resulting optimal geometry comparing both optimisation cases and the datum has been presented in the next section

A quantitative assessment of the results is given in Table 1 and Figure 13. The stress values given here are the actual computed values for the stress, and not the predicted values from the response surface, as is the case in Figure 12.

The stress values were recomputed for the optimal geometry using SimSolid, with the purpose of demonstrating 
that the response surface method was accurate enough to ensure that constraining the predicted stress resulted in constraining the actual stress to the desired value.

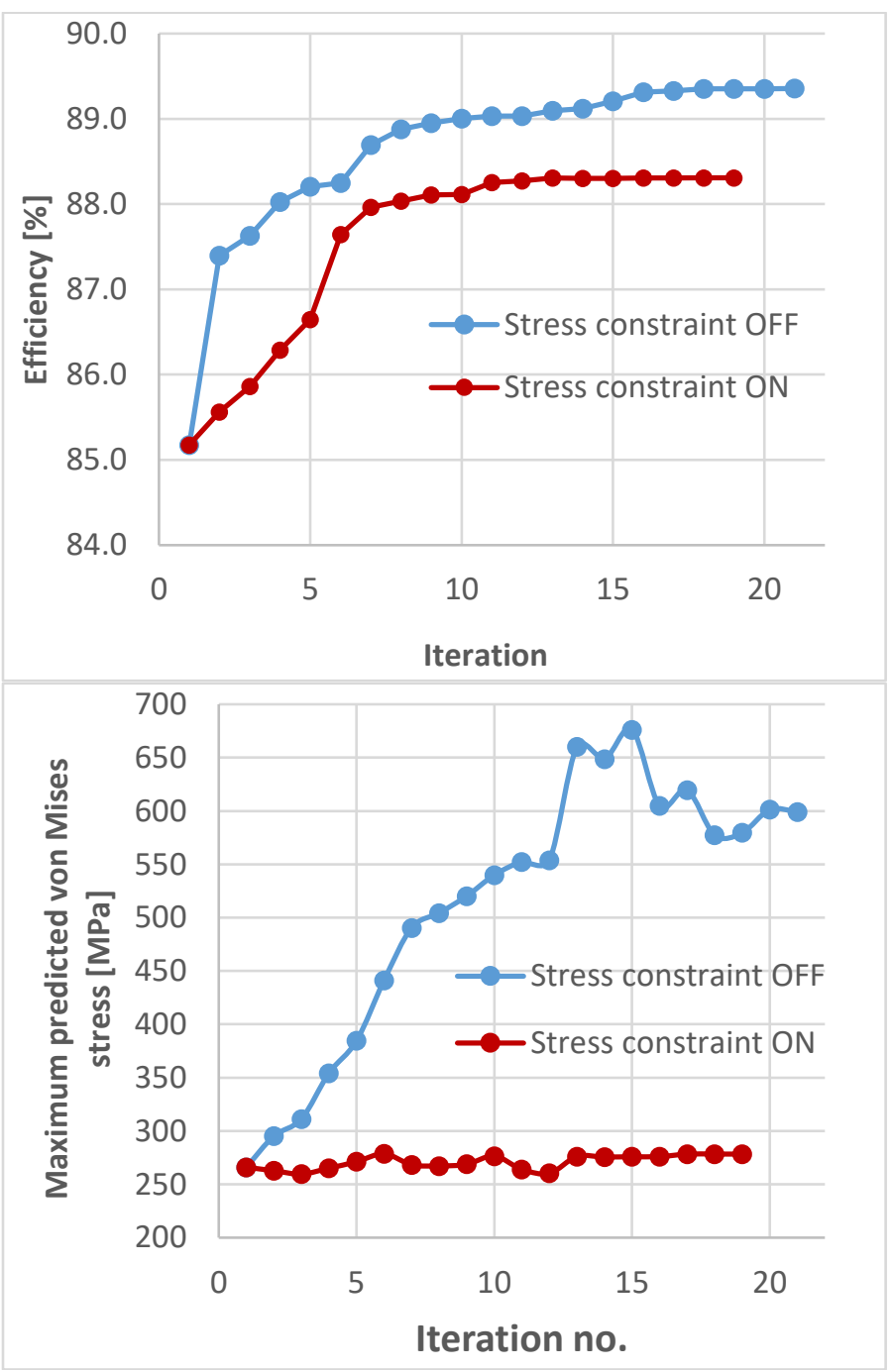

FIGURE 12: UNCONSTRAINED AND STRESS CONSTRAINED OPTIMISATION HISTORIES FOR R37

From this data it can be seen that a considerable increase in efficiency is achieved in the unconstrained optimisation process. The efficiency benefit of the unconstrained optimisation is around $4 \%$. However, the maximum von Mises stress for this case exceeds $500 \mathrm{MPa}$, being more than double the datum value. Although the efficiency benefit drops by approximately $1 \%$, to about $3 \%$, with the stress constraint, the maximum stress is kept within the limit of the datum value, under $250 \mathrm{MPa}$.

Similar efficiency gains were reported in [9], with a maximum value slightly over $3.5 \%$ for the unconstrained optimisation and just under $3 \%$ for the full thickness constrained case. However, a stress analysis based on the same setup described above and conducted on the optimal geometry with thickness constraint from [9] showed that, although the thickness is preserved, the maximum von Misses stress also increases to a value over $500 \mathrm{MPa}$ in the hub fillet region. This shows that constraining the thickness is not sufficient for maintaining the structural integrity of the compressor blades and underlines the importance of a stress constraint.

TABLE 1: OPTIMISATION RESULTS

$\begin{array}{ccc}\text { Geometry } & \text { Efficiency } & \text { Computed maximum } \\ \text { von Mises stress }[\mathrm{MPa}]\end{array}$

\begin{tabular}{l|c|c|}
\hline Datum & 85.18 & 243.8 \\
\hline $\begin{array}{l}\text { Optimal with no } \\
\text { stress constraint }\end{array}$ & 89.36 & 511.8 \\
\hline $\begin{array}{l}\text { Optimal with } \\
\text { stress constraint }\end{array}$ & 88.3 & 238.1 \\
\cline { 2 - 3 }
\end{tabular}

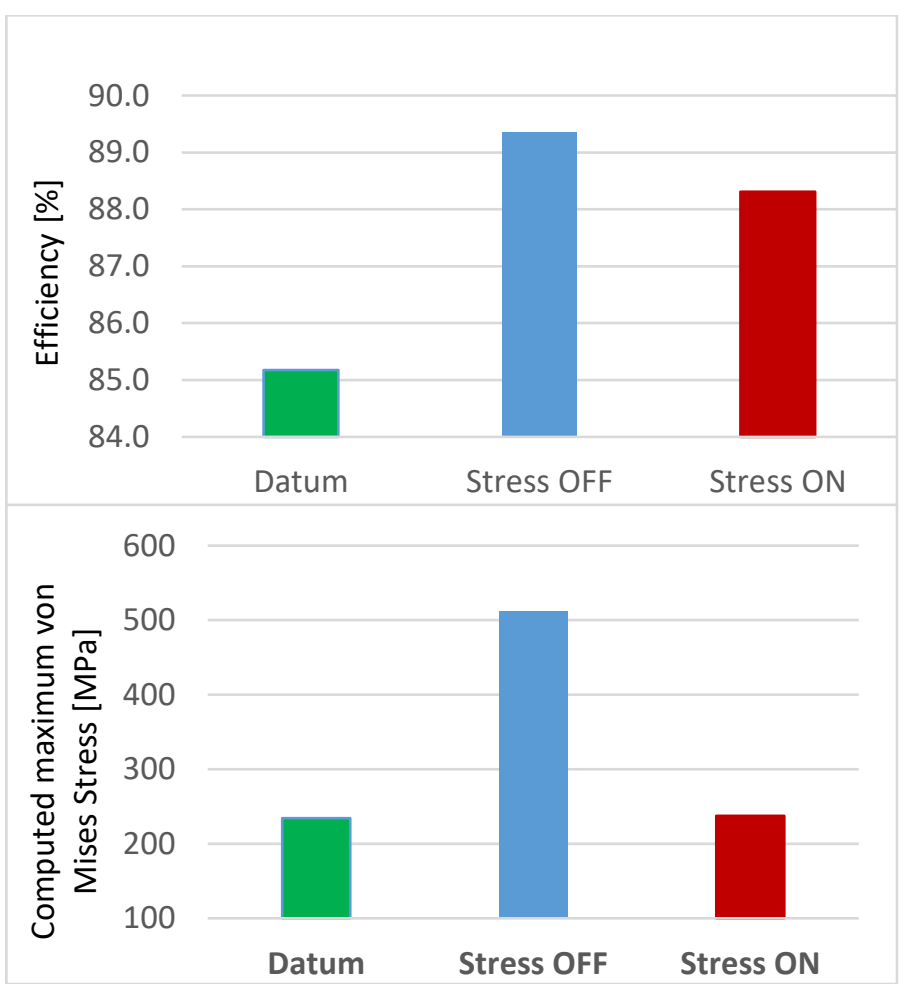

FIGURE 13: OPTIMISATION RESULTS COMPARISON

\subsection{Optimal geometry discussion}

The geometry and flow features for the datum (a) and the optimal design without (b) and with stress constraint (c) are given in Figures 14 to 17. Figure 14 gives the pressure distribution on the suction side along with the regions of zero axial velocity, indicative of flow separation, in grey. Figure 15 details the flow at mid-span by means of relative Mach number, while Figure 16 near the tip. Figure 17 shows the corresponding blade profiles superimposed, at mid-span (up), and the 3D blades (bottom), for comparison purposes. 
static pressure

$E^{1.500 e+05}$

$1.125 \mathrm{e}+5$

75000

$=37500$

$E_{0.000 e+00}$ a)

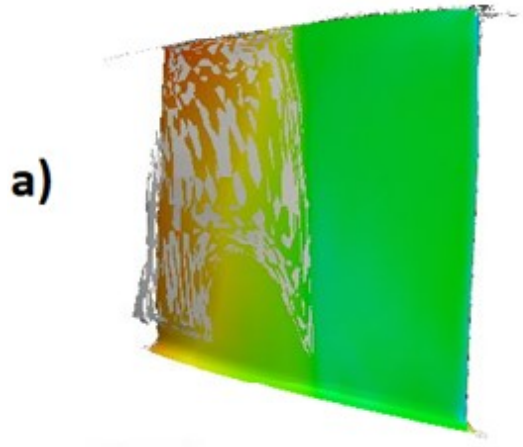

b)

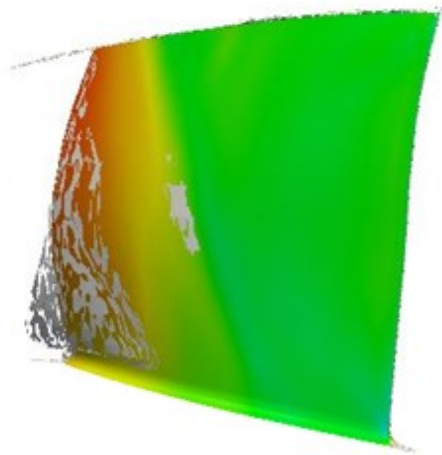

c)

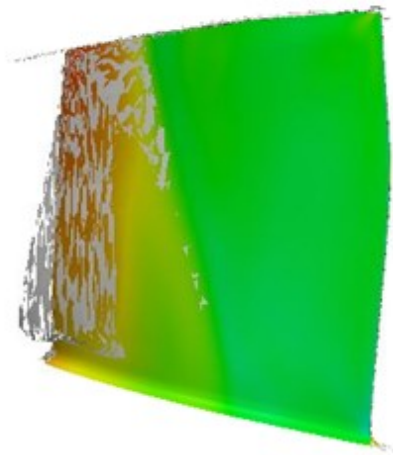

\section{Flow direction}

FIGURE 14: FLOW COMPARISON FOR: a) DATUM, b) OPTIMAL WITH NO STRESS CONTRAINT; c) OPTIMAL WITH STRESS CONSTRAINT

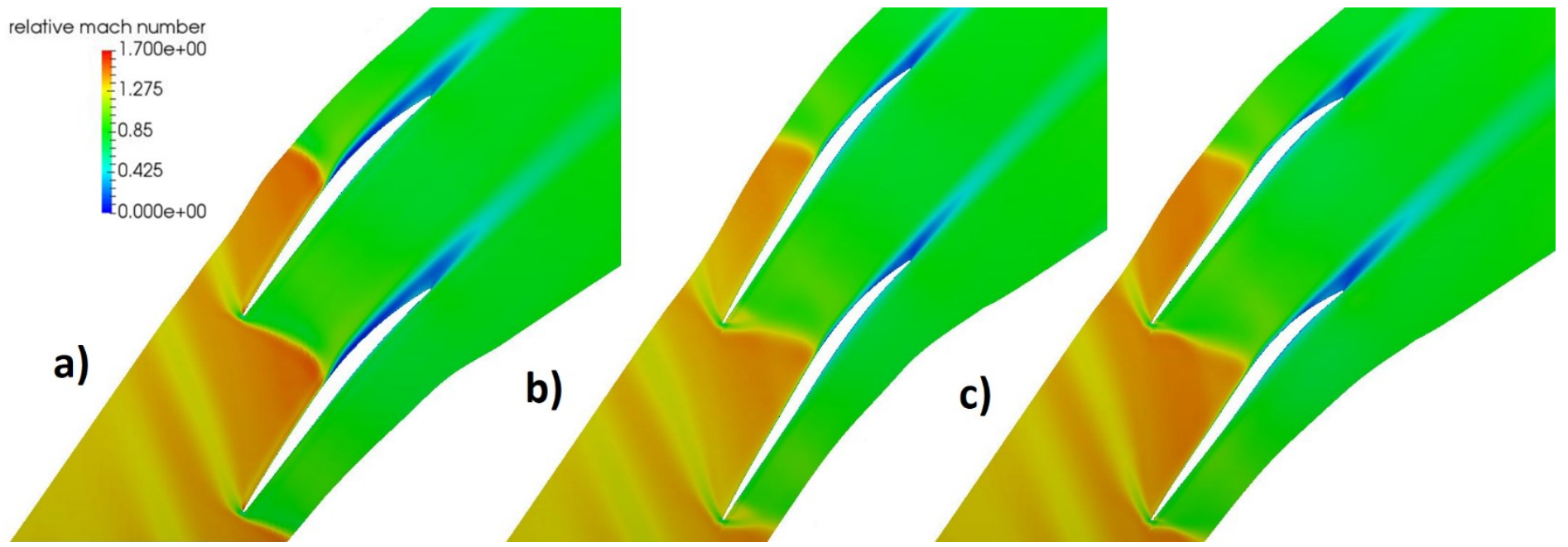

FIGURE 15: MACH NUMBER DISTRIBUTION AT MID-SPAN

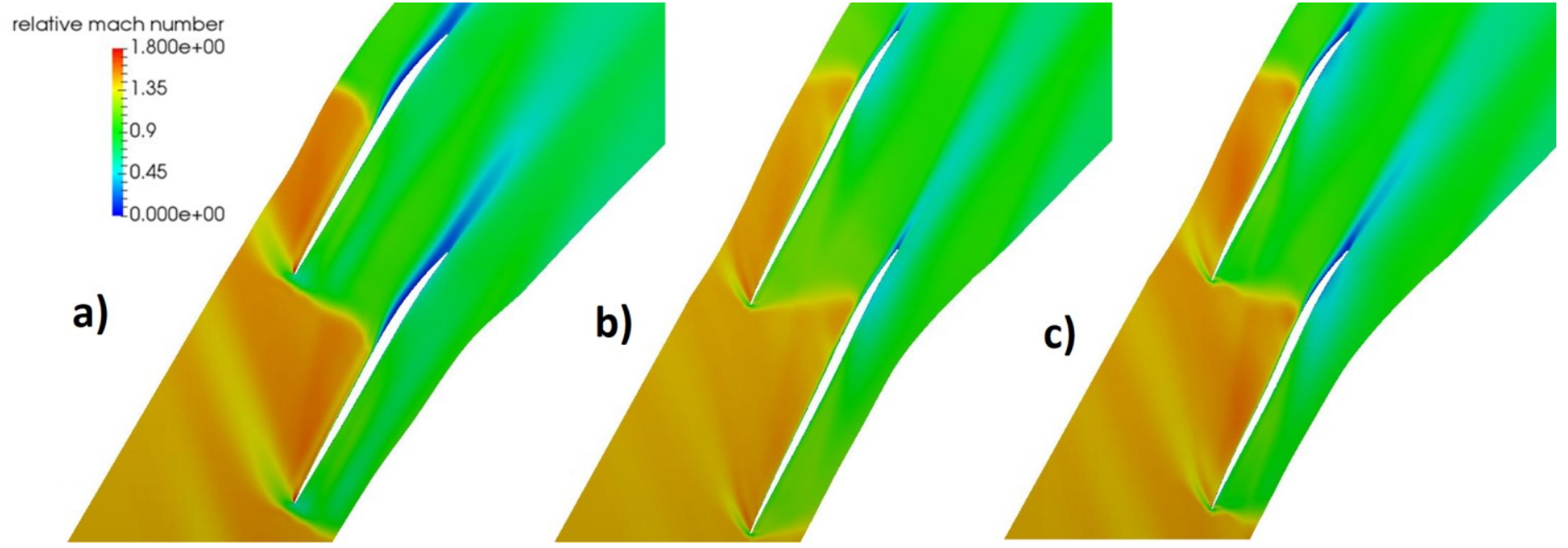

FIGURE 16: MACH NUMBER DISTRIBUTION NEAR TIP 
The datum geometry of Rotor 37 presents a strong shock wave which interacts with the boundary layer and causes the flow to separate (as it can been seen in Figure 14a) and form a large wake, visible in Figures 15a and 16a.

The optimised geometry delays the shock wave and separation from mid-span to the tip of the blade for both optimal designs (Figure 14 to $16, \mathrm{~b}$ and $\mathrm{c}$ ), though this effect is more pronounced for the unconstrained case (Figure 14 to 16, b). At the same time, the Mach number before the shockwave is reduced, further decreasing the losses caused by the shock wave. This is consistent with the previous results reported in the literature $[2,9]$.

From Figure 17, it can be observed that the mid-span section of the unconstrained optimal design is modified by being moved in the circumferential direction, leading to a lean of the blade, combined with a trailing edge recambering. By comparison with the datum, most of the efficiency benefit from the unconstrained optimisation is due to the s-shape of the airfoil, based on the mechanism identified in [2]. The s-shape protrudes into the passage on the suction side, around the shock region and causes a "pre-compression ramp" that reduces slightly the velocity and increases the pressure. Similar features have been observed for the tip section.

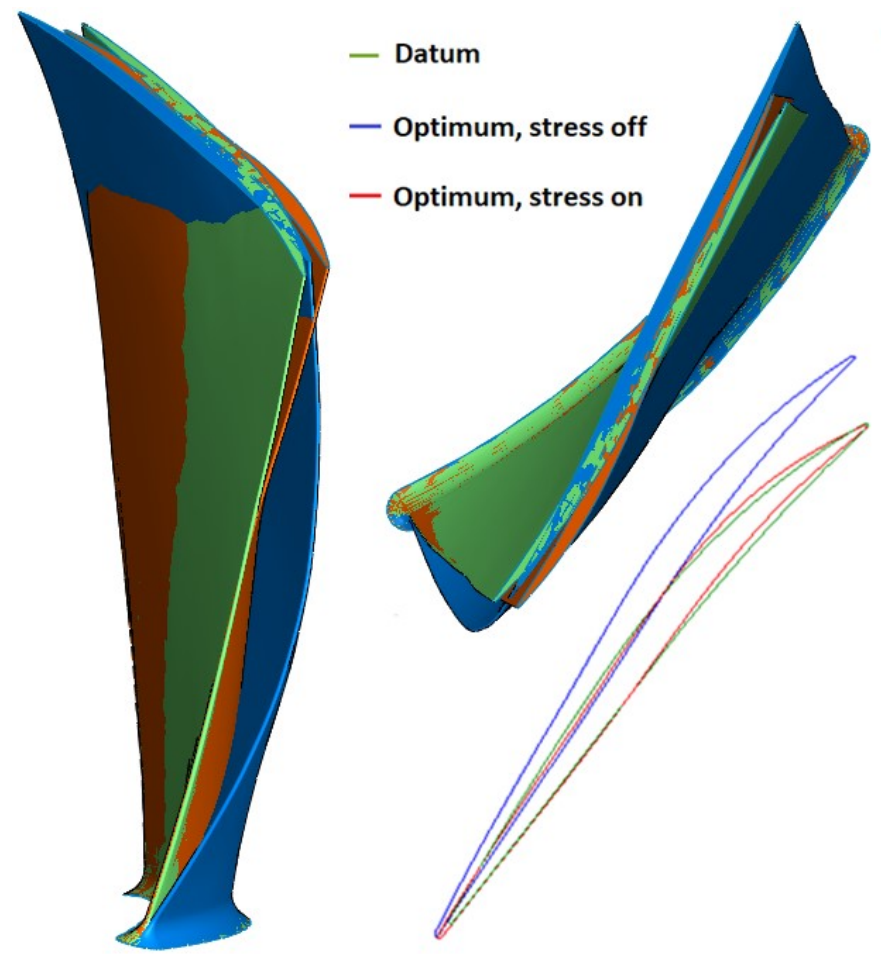

FIGURE 17: 3D BLADES AND MID SPAN BLADE PROFILES

The pressure ratio was not constrained for this study and the optimal solution of the stress constrained optimisation process has a pressure ratio with $1.4 \%$ higher than the datum.

Figure 18 shows the stress distribution on the blade suction side for comparison, for: a) the datum geometry, b) the unconstrained optimal design and c) the constrained optimal design, using the same plot legend, limited to $250 \mathrm{MPa}$.

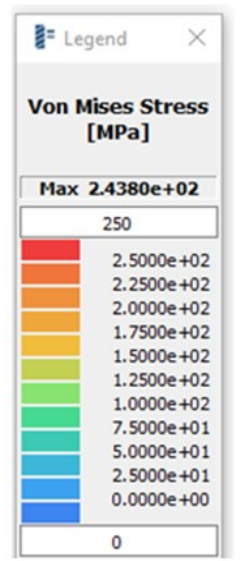

b)

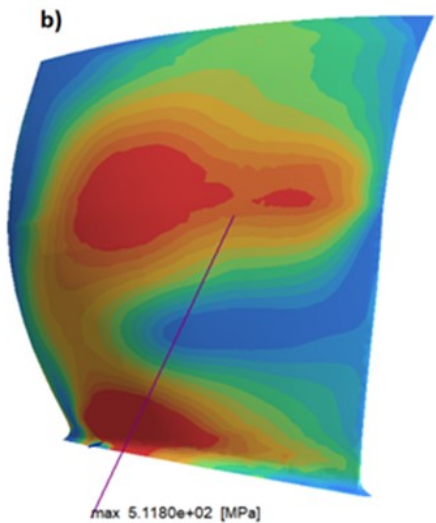

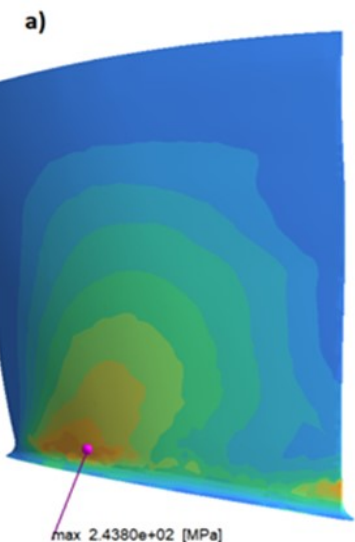

c)

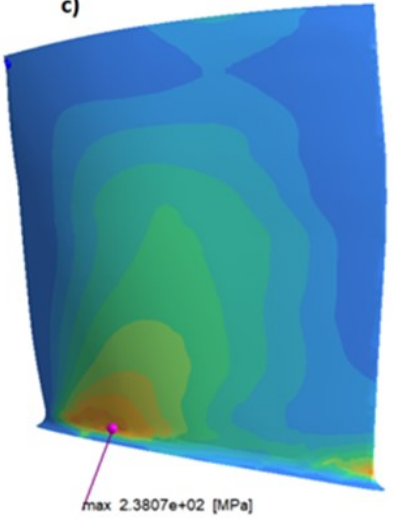

FIGURE 18: VON MISES STRESS DISTRIBUTION (SUCTION SIDE) FOR: a) DATUM, b) OPTIMAL WITH NO STRESS CONTRAINT; c) OPTIMAL WITH STRESS CONSTRAINT

The optimal geometry with stress constraint has a similar stress distribution to the datum, with a close value for the maximum von Misses stress. The lean introduced in the unconstrained optimal geometry causes a major stress increase. The stress value exceeds $250 \mathrm{MPa}$ on almost half of the suction surface.

The maximum stress value for this geometry is slightly over $500 \mathrm{MPa}$ and has moved from the suction side hub fillet, where it is located for the datum and constrained optimal geometry, to the mid-section of the pressure side. The change in the location of the maximum stress, visible in Figure 19, is a consequence of the blade lean. This can produce a bending moment due to the tangential component of the centrifugal force, which can, in turn, lead to a local increase of the equivalent stress. At the same time, rotor blades are usually tapered both in chord and in thickness from root to tip. For this case, the blade exhibits a large chord at the tip, while being reduced towards the mid-section. The effect is a distribution of the stress similar to a dog-bone-shaped specimen under tensile stress, where the maximum stress is located at the mid-section. 
Based on the flow and stress analyses, it can be concluded that the constrained optimal design loses the potential efficiency gain in favour of structural integrity.

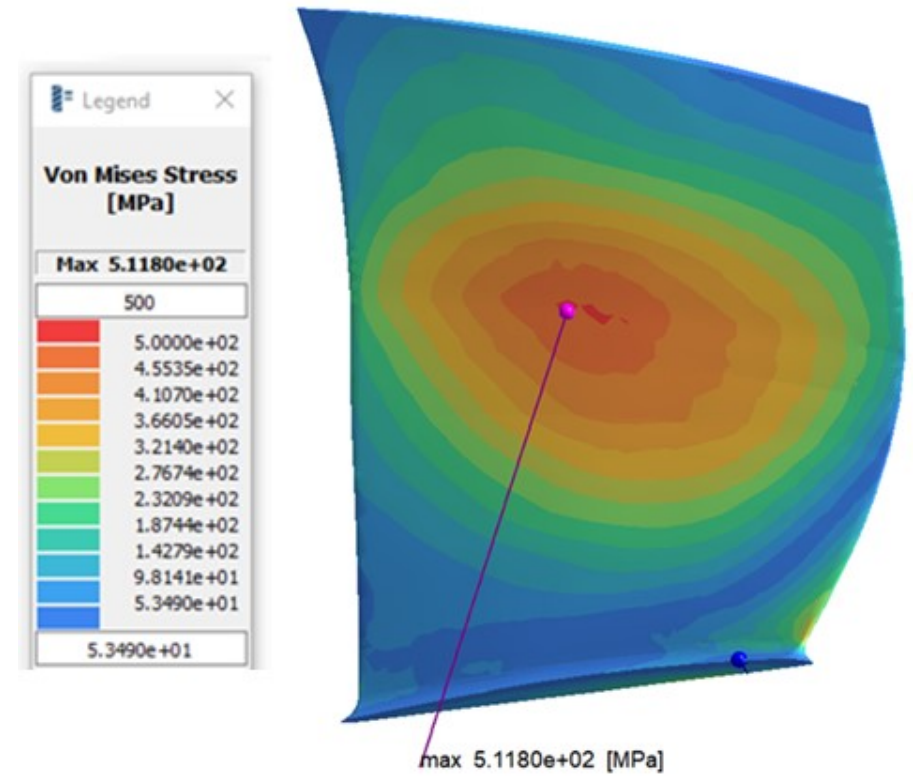

FIGURE 19: VON MISES STRESS FOR OPTIMAL DESIGN WITH STRESS CONSTRAINT (PRESSURE SIDE)

\section{CONCLUSION}

This work successfully applied a structurally constrained aerodynamic adjoint based optimisation method to a highly loaded compressor blade. The parametrisation of the geometry was done using free-form deformation, for increased flexibility in the design.

The optimisation process had the aerodynamic efficiency as objective function and maximum equivalent (von Mises) stress as constraint. The aerodynamic optimisation relies on the computation of gradients using a discrete adjoint approach, while the structural constraint was resolved by using a response surface and finite differences, both integrated with a sequential least squares programming algorithm.

Several stress response surfaces were compared based on the coefficient of determination and the root-mean-square error to select the most suitable one. Based on the results of two different samplings of the design space, it was concluded that the 20,000 points polynomial response surface gives the best results. This response surface was further used as a surrogate model in the structurally constrained aerodynamic adjoint optimisation of the highly loaded compressor blade.

It was demonstrated that without a structural constraint, the maximum stress on the blade increases drastically. Although some of the potential efficiency gain was sacrificed in the favour of structural integrity, the tool successfully improved the Rotor 37 efficiency by over 3\%, while maintaining the maximum von Mises stress under the datum value. This shows the effectiveness of the method in increasing the efficiency of compressor blades while maintaining a low value for the maximum von Mises stress and the importance of using structural constraints early in the optimisation process.

The methodology presented here can be extended to other turbomachinery applications for aerodynamic optimisations with structural constraints at low computational costs while using a large number of design parameters.

\section{ACKNOWLEDGEMENTS}

This project has received funding from the European Union's Horizon 2020 research and innovation programme under grant agreement No 769025. The authors would like to thank Rolls-Royce for their support and permission to publish the work.

\section{REFERENCES}

[1] Reid, L., and Moore, R. D., 1978, "Performance of SingleStage Axial-Flow Transonic Compressor With Rotor and Stator Aspect Ratios of 1.19 and 1.26, Respectively, and With Design Pressure Ratio of 1.82", NASA Lewis Research Center, Cleveland, OH, Report No. NASA-TP-1338.

[2] John, A., Shahpar, S., and Qin, N., 2017, "Novel compressor blade shaping through a free-form method", Journal of Turbomachinery, 139(8), pp. 081002.

[3] Chicane C., 2018, "Multidisciplinary Design Optimisation of Aero-Engine Fan Blades", Ph.D. dissertation, University of Cambridge, UK.

[4] Ahn, C.S., and Kim, K.Y., 2003, "Aerodynamic design optimization of a compressor rotor with Navier-Stokes analysis", Journal of Power and Energy, 217, pp. 179-183.

[5] Benini, E., 2004, "Three-Dimensional Multi-Objective Design Optimization of a Transonic Compressor Rotor", J. Propul. Power, 20(3), pp. 559-565.

[6] Polynkin, A., Toropov, V., and Shahpar, S., 2010, "Multidisciplinary Optimization of Turbomachinery Based on Metamodel Built by Genetic Programming", AIAA Paper No. 2010-9397.

[7] Shahpar, S., Polynkin, A., and Toropov, V., 2008, "Large Scale Optimization of Transonic Axial Compressor Rotor Blades", AIAA Paper No. 2008-2056-891.

[8] Duta, M. C., Shahpar, S., and Giles, M. B., 2007, "Turbomachinery Design Optimization Using Automatic Differentiated Adjoint Code", ASME Paper No. GT200728329.

[9] John, A., Qin, N., and Shahpar, S., 2020, “The Influence of Parameterisation Setup on the Constrained Adjoint Optimisation of Transonic Fan Blades", ASME Paper No. GT2020-15352.

[10] Sederberg, T.W., and Parry, S.R., 1986, "Free-form deformation of solid geometric models", Proceedings of the 13th Annual Conference on Computer Graphics and Interactive Techniques - SIGGRAPH '86, pp. 151-160. 
[11] Shahpar, S., and Caloni, S., 2013. "Adjoint optimisation of a high-pressure turbine stage for a lean-burn combustion system", Proceeding of 10th ETC, Lappeenranta, Finland.

[12] Li, L., Jiao, J., Sun, S., Zhao, Z., and Kang, J., 2019, "Aerodynamic shape optimization of a single turbine stage based on parameterized free-form deformation with mapping design parameters", Energy, 169, pp. 444-455.

[13] Economon, T., Palacios, F., and Alonso, J., 2012, "Optimal shape design for open rotor blades", In 30th AIAA Applied Aerodynamics Conference, pp. 3018.

[14] Jameson, A., Pierce, N., and Martinelli, L., 1998, "Optimum aerodynamic design using the navier-stokes equations", J. Theor. Comp. Fluid Mech., 10, pp. 213-237.

[15] Müller, L., Verstraete, T., 2017, "CAD Integrated Multipoint Adjoint-Based Optimization of a Turbocharger Radial Turbine", Int. J. Turbomach. Propuls. Power, 2(3), pp. 14.

[16] Vitale, S., Pini, M., and Colonna, P., 2020, "Multistage Turbomachinery Design Using the Discrete Adjoint Method Within the Open-Source Software SU2", J. Propulsion and Power, 36(3).

[17] Russo, V., Orsenigo, S., Mueller, L., Verstraete, T., and Lavagnoli, S., 2019, "Adjoint based aerodynamic optimization of a multi-splitter turbine vane frame", ASME Paper No. GT2019-91608.

[18] Luo, J., and Zheng, Y., 2019, "Aerodynamic shape optimization of a turbine blade considering geometric uncertainty using an adjoint method", ASME Paper No. GT2019-90051.

[19] Liefke, A., Marciniak, V., Backhaus, J., Frey, C., Gottschalk, H., and Janoske, U., 2019, “Aerodynamic impact of manufacturing variation on a nonaxisymmetric multi-passage turbine stage with adjoint CFD”, ASME Paper No. GT201991480.

[20] Torreguitart, I.S., Verstraete, T., and Mueller, L., 2018, "Optimization of the LS89 Axial Turbine Profile Using a CAD and Adjoint Based Approach", Int. J. Turbomach. Propuls. Power, 3(3), pp. 20.

[21] Rubino, A., Pini, M., Colonna, P., Albring, T., Nimmagadda, S., Economon, T., and Alonso, J., 2018, "Adjoint-based fluid dynamic design optimization in quasiperiodic unsteady flow problems using a harmonic balance method", Journal of Computational Physics 372, pp. 220-235.

[22] Vasilopoulos, I., Flassig, P., and Meyer, M., 2016, "Aerodynamic Optimization of the TurboLab Stator: A Comparative Study between Conventional and Adjoint based Approaches", International Conference on Numerical Optimisation Methods for Engineering Design (NOED), TU Munchen, Germany.

[23] Verstraete, T., Müller, J.D., Müller, L., 2017, “CADBased Adjoint Optimization of the Stresses in a Radial Turbine", ASME paper No. GT2017-65005

[24] Schwalbach, M., Verstraete, T., and Gauger, N.R., 2019, "Discrete adjoint gradient evaluations for linear stress and vibration analysis", Computing and Visualization in Science, 21, pp. 23-31.
[25] Verstraete, T., Müller, J.D., Müller, L., 2017 "Multidisciplinary Adjoint Optimization of Turbomachinery Components Including Aerodynamic and Stress Performance", 35th AIAA Applied Aerodynamics Conference, AIAA Paper No. AIAA 2017-4083.

[26] Müller, L., Verstraete, T., and Schwalbach, M., 2019, "Adjoint-based Multidisciplinary, Multipoint Optimization of a Radial Turbine considering Aerodynamic and Structural Performances", ASME Paper No. GT2019-91823.

[27] Muller, L., Verstraete, T., 2019, “Adjoint-Based MultiPoint and Multi-Objective Optimization of a Turbocharger Radial Turbine", Int. J. Turbomach. Propuls. Power, 4(2), pp. 10.

[28] Luo, J., Zhou, C., Liu, F., 2014, "Multipoint design optimization of a transonic compressor blade by using an adjoint method", Journal of Turbomachinery 136(5), pp. 051005

[29] Shahpar, S., and Lapworth, L., 2003, "PADRAM: Parametric Design and Rapid Meshing System for Turbomachinery Optimisation," ASME Paper No. GT200338698.

[30] Lapworth, L., 2004, "Hydra-CFD: a framework for collaborative CFD development", International Conference on Scientific and Engineering Computation (IC-SEC), Singapore, Vol. 30.

[31] Dunham, J., 1998, "CFD validation for propulsion system components (La validation CFD des organes des propulseurs), Tech. rep., DTIC Document.

[32] Giles, M. B., Duta, M. C., Muacute, J.-D., and Pierce, N. A., 2003, "Algorithm developments for discrete adjoint methods", AIAA journal, 41(2), pp. 198-205.

[33] Apanovitch, V., 1991, "The method of external finite element approximations" Minsk, ISBN 5-339-00597-6

[34] Zienkiewicz, O.C., and Zhu, J.Z., 1987, "A Simple Error Estimator and Adaptive Procedure for Practical Engineering Analysis", International Journal for Numerical Methods in Engineering, 24, pp. 337-357.

[35] Moore, D. S., 1991, "Statistics: Concepts and Controversies", 3rd ed., 439 pp., W. H. Freeman, New York.

[36] Oliphant, T.E., 2007, "Python for scientific computing", Computing in Science \& Engineering, 9(3), pp. 10-20. 